\title{
Programa de intervenção motora para escolares com indicativo de TRANSTORNO DO DESENVOLVIMENTO DA COORDENAÇÃO - TDC
}

M OTOR INTERVENTION PROGRAM FOR SCHOOL CHILDREN WITH SIGNS OF DEVELOPMENTAL COORDINATION DISORDER - DCD

\author{
Eva Vilma Alves da SILVA ${ }^{1}$ \\ Andressa Ribeiro CONTREIRA 2 \\ Thaís Silva BELTRAME ${ }^{3}$ \\ Fabiana Flores SPERANDIO ${ }^{4}$
}

RESUM O: este estudo objetivou verificar os efeitos de um programa de intervenção motora para escolares com indicativo de Transtorno do Desenvolvimento da Coordenação. Participaram do estudo seis escolares na faixa etária de 10 anos, do gênero feminino e masculino, matriculados em uma escola municipal no interior do Estado de Santa Catarina. A avaliação motora foi mensurada por meio do M ovement A ssessement Battery for Children (MABC-2). $O$ teste abrange as faixas etárias de três a 16 anos dentro de cada faixa etária são agrupadas oito tarefas em três categorias de habilidades: destreza manual, Iançar e receber, equilíbrio. As intervenções foram baseadas na abordagem da Educação Física Desenvolvimentista em ambiente escolar. As sessões foram real izadas individual mente com 20 sessões de intervenção motora para cada escolar, num total de 120 sessões, com frequência de duas aulas semanais e com duração de 45 minutos. Para interpretação dos dados foi utilizado o teste Wilcoxon no pacote estatístico SPSS 13.0 for Windows. Os resultados evidenciaram diferenças estatisticamente significativas após a intervenção motora $(p<0,05)$. Em relação aos resultados do desempenho motor por habilidade, verificou-se que a habilidade equilíbrio foi a que apresentou melhor resultado após a intervenção, revelando que uma proposta de intervenção motora foi eficaz para a melhora no desempenho motor das crianças com indicativos de TDC. A través deste estudo evidenciou-se os benefícios da participação de escolares com problemas motores em programas de intervenção, no sentido de monitorar o seu progresso em termos de desenvolvimento motor, visando à mel hora das dificuldades de movimento tanto nas habilidades esportivas quanto nas atividades diárias.

PA LA V RA S - CH A VE: Intervenção Pedagógica. Desenvolvimento Motor. Habilidade M otora. Transtorno do Desenvolvimento da Coordenação.

\footnotetext{
${ }^{1}$ Mestranda em Ciências do Movimento Humano pela Universidade do Estado de Santa Catarina - UDESC. evameeg@ig.com.br

${ }_{2}^{2}$ Mestranda em Ciências do Movimento Humano pela Universidade do Estado de Santa Catarina - UDESC. andressa_contreira@yahoo.com.br

${ }^{3}$ Doutora em Ciências do Movimento Humano, Professora do Centro de Educação Física e Fisioterapia da Universidade do Estado de Santa Catarina (UDESC) e Diretora de Ensino da Universidade do Estado de Santa Catarina. Correspondência: Universidade do Estado de Santa Catarina, LADADE - Laboratório de Distúrbios da A prendizagem e do Desenvolvimento. bhthais@terra.com

${ }^{4}$ Doutora em Engenharia de Produção e Sistemas, Professora do Centro de Educação Física e Fisioterapia da Universidade do Estado de Santa Catarina (UDESC), Coordenadora do estágio em Fisioterapia e Educação Física na UDESC. Correspondência: Universidade do Estado de Santa Catarina, LADADE - Laboratório de Distúrbios da A prendizagem e do Desenvolvimento, d2fs@udesc.br
} 
ABSTRACT : This study aimed to verify the effects of a motor intervention program for students with signs of DCD. Six 10 year-old students, both male and female, who were registered at a municipal school in the interior of the state of Santa Catarina participated in the study. Motor capacity was measured using the Movement Assessment Battery for Children (MABC-2), one of the most commonly used instruments for assessing children's movement difficulties. The test encompasses the age groups of 3 to 16 years; for each age group, eight tasks are grouped into three skill categories: manual skill, throw and catch, balance. The interventions were based on the Developmental Physical Education approach in school environments. The sessions were doneindividually with 20 motor intervention sessions for each student, in a total of 120 sessions, frequency of two weekly classes with 45 minutes duration. The Wilcoxon test with the statistical package SPSS 13.0 for Windows was used for data interpretation. The results showed statistically significant differences after motor intervention ( $p<0,05)$. Regarding theresults of motor skill performance, it was found that balance skills presented better improvement results after intervention, revealing that a motor intervention proposal was effective with children with signs of DCD. This study revealed the importance of promoting students with motor disabilities participation in intervention programs in order to monitor their motor development progress, so as to improve movement difficulties both in sports and in daily activities.

KEYWORD S: Special Education. Rehabilitation Program. Motor Development.

\section{INTRODUÇÃO}

O desenvolvimento motor é uma alteração contínua no comportamento motor ao longo da vida, proporcionada pela interação entre as necessidades da tarefa, abiologia do indivíduo eas condições do ambiente, que, sebem relacionados, favorecem o surgimento de novas formas de execuções motoras das crianças. Contudo, em casos de alteração em algum deles, o processo de desenvolvimento pode ser colocado em risco (SANTOS; DANTAS; OLIVEIRA, 2004; GALLAHUE; OZMUN , 2005).

Em se tratando de fatores de risco para o desenvolvimento infantil, pode-se destacar a existência dealguns déficits, tanto da aprendizagem quanto do desenvolvimento motor, como o Transtorno do Desenvolvimento da Coordenação (TDC). OTDC ocorrequando há atraso no desenvolvimento de habilidades motoras ou dificul dades para coordenar os movimentos, queresultam em incapacidade da criança para desempenhar atividades diárias, estimando-se uma prevalência do transtorno em $5 \%$ a $15 \%$ das crianças (MISSIUNA, 2003; RUIZ et. al., 2003). As crianças com indicativo de TDC, sem qualquer dano neurológico ou fisiológico aparente, ao realizar simples tarefas motoras do dia-a-dia tais como abotoar uma camisa, usar o garfo e a faca, amarrar o sapato, têm propensão a deixar cair às coisas, são desajeitadas, demonstram fraco desempenho escolar, nos esportes e nas atividades cotidianas. Cabe destacar que al gumas crianças podem apresentar dificuldades na realização de todas as habilidades motoras, e até mesmo na fala que não é clara e fluente (FERREIRA et al., 2006).

Devido à alta prevalência da dificuldade motora apresentada e a importância que os aspectos do movimento humano possuem sobre o cotidiano de cada pessoa, pesquisadores têm se dedicado às investigações de indivíduos com déficit na coordenação motora (CERMAK, 1985; GEUZE, 2001; MISSIUNA, 2003; JORGE, 2003; SANTOS; DANTAS; OLIVEIRA, 2004; FERREIRA et al., 2006; PELLEGRINI et al., 2006; FRANÇA, 2008; LEMOS; FRACAROLI; ROSA, 2009; 
SILVA, 2009; MIRAN DA , 2010) suas possíveis causas (JORGE, 2003; FERREIRA et al., 2006), padronização de instrumentos existentes para identificação (GEUZE, 2001; HENDERSON; SUGDEN; BARNETT, 2007), programas de intervenção motora (VALENTINI, 2002; POETA; ROSA NETO, 2005; MULLER, 2008; PICK, 2008), entre outras ações.

A intervenção motora é indicada para indivíduos com necessidades especiais ou déficit motor; sua finalidade, seguindo uma abordagem desenvolvimentista, deveatender as principais necessidades do aluno, promovendo a interação dinâmica entreas características do executante, da tarefa edo ambiente, objetivando o aumento do repertório motor (GALLAHUE; OZMUN, 2005). A identificação de fatores deficitários através da avaliação do perfil motor énecessária para traçar diretrizes de intervenção direcionadas à população em questão. Dessa forma, com base no perfil das crianças, podem ser elaborados programas de educação ou reeducação motora, visando proporcionar a coordenação e ritmo e tornar o cérebro da criança um órgão com maior capacidade para captar, integrar, armazenar, elaborar e expressar informações (CAMPOS et al., 2008).

Em relação às estratégias de intervenção, diversos estudos têm sido realizados com o intuito de verificar sua influência na melhoria das habilidades motoras em crianças em fase escolar identificadas com al guma dificul dade motora (VALENTINI, 2002; CANTELL; SMYTH; AHONEN, 2003; MISSIUNA, 2003; POETA; ROSA NETO, 2005; HENDERSON ; SUGDEN ; BA RNETT, 2007; PIFFERO, 2007; MULLER, 2008; PICK, 2008; ROSA et al., 2008; PEREIRA; SILVA; CAMPOS, 2008; MEDINA, 2008; CAMPOS et al., 2008; TSAI, 2009; BRAGA et al., 2009; CONTREIRA et al., 2010), cognitiva (CAMPOS et al., 2008; TSAI, 2009) ou social (VALENTINI, 2002; PIFFERO, 2007; PICK, 2008).

Diante da relevância dos programas de intervenção motora para o desenvolvimento de crianças com dificuldades motoras econsiderando a existência depoucos estudos deintervenção motora com enfoque no TDC, o presente estudo objetivou verificar os efeitos de um programa de intervenção motora no desenvolvimento motor de escolares com indicativo de transtorno do desenvolvimento da coordenação motora.

\section{MÉTOdo}

Este estudo se caracteriza como quase experimental, pois estabelece uma relação de causa e efeito. Isto é, uma variável independente é manipulada para julgar seu efeito sobre a variável dependente (THOMAS; NELSON, 2002).

A pesquisa foi realizada em uma escola municipal de Florianópolis/ SC, na qual o Laboratório de Distúrbios da A prendizagem e do Desenvolvimento (LADADE) vem desenvolvendo estudos desde2005, com a finalidade de identificar a presença de transtornos de comportamento, dificuldades de movimento, bem como propor estratégias de intervenção motora. 
Em uma pesquisa do mesmo laboratório, realizada por Silva (2009), foram avaliadas 406 escolares com idades de sete a 10 anos, e destes, 45 foram identificados com dificuldades motoras. Diante disso, os próximos trabalhos realizados buscaram realizar programas de intervenção motora para pequenos grupos de escolares, já que esta era um proposta nova para a escola e para os pesquisadores que vinham avaliando essas crianças.

Nesse contexto, dos 45 escolares que apresentavam o indicativo de dificuldade, 15 foram convidados a participar do programa de intervenção, houve uma perda amostral devido a motivos de saúde, tais como a gripe $\mathrm{H} 1 \mathrm{~N} 1$, bem como o não consentimento dos pais. Diante disso, o grupo de participantes desta pesquisa foi constituído por seis escolares, na faixa etária de 10 anos, de ambos os gêneros, matriculados regularmente em uma escola municipal de Florianópolis/ SC. Como critério deinclusão foi considerado o interesse dos escolares em participar do estudo, a inexistência de deficiências que impedissem a realização das tarefas da avaliação motora e ter o termo de consentimento livre e esclarecido assinado pelo responsável.

Esta pesquisa foi realizada de acordo com a resolução no 196/ 96 do Consel ho Nacional de Saúde (CNS) e aprovada em seus aspectos éticos e metodológicos pelo ComitêdeÉtica ePesquisa em Seres Humanos da Universidade do Estado de Santa Catarina (CEPSH/ UDESC), sob protocolo nำ130/ 2009.

\section{1 Procedimentos de intervenção}

O programa de intervenção foi baseado na abordagem da Educação Física Desenvolvimentista cujo objetivo écentrado no indivíduo, nas circunstâncias ambientais e nos objetivos da tarefa, envolvendo aquisição e refinamento de movimentos ecrescentecompetência física (GALLA HUE; DON NELLY, 2008). Cabe destacar que as sessões interventivas foram planejadas conforme as prioridades de dificuldades de movimentos apresentada por cada escolar quando avaliado motoramentepel o testeMABC-2. As mesmas foram realizadas em ambienteescolar, utilizando os espaços de sala de aula e pátio. Como material pedagógico foram utilizados balões, bil boquê, cesta de basquete, arcos, bolas, raquete, cordas, jornais, boliche, barbante, canudos, massinha de modelar, formas geométricas, jogos pedagógicos que exigem raciocínio rápido, entre outros.

O programa enfatizou as habilidades amplas, finas e combinadas tais como rebater um balão com uma raquete utilizando todo o espaço do ambiente para deslocar; saltar se deslocand o com uma bola entre os pés; pular corda; lançar uma bola dentro do arco; caminhar passando o corpo por dentro do arco; lançar uma bola no cesto; jogo do boliche; fazer canudos com jornais; fazer objetos com massinha de modelar; passar o barbante em canudos cortados em pequenos pedaços; jogos pedagógicos e brinquedos que exigem raciocínio rápido, percepção de espaço e tempo; encaixe e desencaixe de formas geométricas, entre outros. As 
atividades desenvolvidas foram desafiadoras, seguiram um processo gradual (simples-complexas), sendo direcionadas às dificuldades motoras apresentadas pelas crianças e identificadas a partir da avaliação motora. Foram realizadas nos horários das aulas deeducação física nos períodos matutino evespertino, constando de20 sessões individuais deintervenção motora, real izadas duas vezes por semana, com duração de 45 minutos, totalizando 120 sessões.

\subsection{Procedimento de coleta de dados}

A aplicação do teste motor foi realizada em ambiente adequado e iluminado em ambas as situações do estudo (prée pós-teste) com duração de 30 a 40 minutos por aluno.

Os escolares foram avaliados individualmente por um avaliador devidamente capacitado para aplicação dos testes motores. A ntes da realização de cada tarefa o avaliador demonstrava ao aluno como deveria proceder e depois o avaliado tinha oportunidade defamiliarização com o instrumento para quedepois dessa familiarização fosse real izada a tentativa a ser registrada na ficha deavaliação. Conforme consta no protocolo do teste, são realizadas as tarefas de habilidades manuais (três tarefas), seguidas de habilidades com bola (duas tarefas) e posteriormente as tarefas de equilíbrio estático e dinâmico (três tarefas).

É importante destacar queos escolares foram orientados a vestir roupas confortáveis e cal çados ad equados para realização das tarefas motoras.

\subsection{ANÁlise dos dados}

Para interpretação dos dados obtidos por meio da avaliação motora foi utilizado o pacote estatístico SPSS 13.0 for Windows. Diante da verificação da não normalidade dos dados, foi utilizado o teste não-paramétrico Wilcoxon para amostras pareadas, comparando o desempenho motor dos escolares no MABC-2 antes e após o programa de intervenção motora.

\section{Resultados}

A Tabela 1 apresenta os resultados do desempenho motor total dos escolares em pré e pós-teste por categoria de habilidades. Os resultados do Teste Wilcoxon evidenciaram diferenças estatisticamente significativas após a intervenção motora $(p<0,05)$, demonstrando que esta foi efetiva para a mel hora do desempenho motor dos escolares com indicativo de TDC. Quanto ao desempenho por categoria dehabilidades, somente a habilidade equilíbrio, apresentou diferença estatisticamente significativa após o programa, contudo, através da observação das médias é possível perceber que ocorreu melhora nas demais habilidades. 
Tabela 1 -Resultado das médias do pré e pós-teste no MABC - 2

\begin{tabular}{cccccccccccccc}
\hline \multicolumn{1}{c}{ Pré-Teste } & \multicolumn{1}{c}{ Pós-Teste } \\
\hline Habilidades & Média & mediana & $\mathrm{dv}$ & Max & min. & Média & mediana & $\mathrm{dv}$ & $\max$ & min. & P \\
DM & 6.16 & 6.00 & 1.16 & 8.00 & 5.00 & 7.16 & 7.50 & 1.94 & 9.00 & 4.00 & 0,269 \\
LR & 8.33 & 8.50 & 2.16 & 11.00 & 5.00 & 9.33 & 9.50 & 2.73 & 13.00 & 5.00 & 0,414 \\
EQ & 5.50 & 6.00 & 2.58 & 8.00 & 2.00 & 10.66 & 11.50 & 2.80 & 14.00 & 6.00 & $0.026^{*}$ \\
$\begin{array}{c}\text { Pontuação } \\
\text { Total do } \\
\text { Teste }\end{array}$ & 54.50 & 59.50 & 11.91 & 66.00 & 38.00 & 72.16 & 73.50 & 10.18 & 87.00 & 58.00 & $0.018^{*}$ \\
\hline
\end{tabular}

Legenda:

DM: destreza manual; LR: lançar e receber; EQ: equilíbrio

*Diferença estatisticamente significativa para $\mathrm{p} \ll 0,05$

Por meio da observação do Gráfico 1, constatou-se a melhora no desempenho motor dos escolares no MABC-2 após a intervenção motora.

Gráfico 1 - Representação do desempenho motor dos escolares no MABC-2em pré e pós-teste

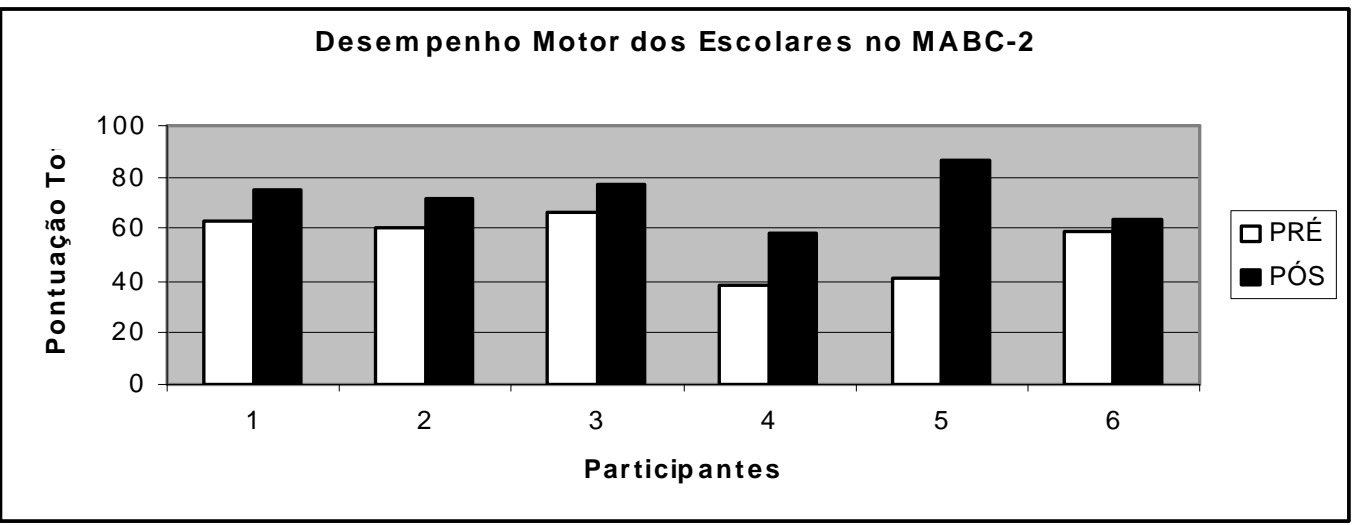

\section{Discussão}

Os resultados do presente estudo vão ao encontro da afirmação de Missiuna (2003) ao colocar que a intervenção motora pode ajudar a criança com 
TDC a progredir ao desempenhar muitas das atividades diárias com mais sucesso, evitando o fracasso e frustrações em práticas de atividades físi cas. Ainda, Cantell et al., (2003) acrescentam que as intervenções preventivas podem evitar efeitos secundários do TDC, como isolamento e o baixo desempenho escolar.

Também foram evidenciados os benefícios da intervenção motora no estudo de revisão bibliográfica realizado por Willrich et al., (2008), no qual foi realizada uma análise de publicações nas bases dedados M edline, Pubmed eScielo, sobre desenvolvimento motor, fatores de risco (ambientais e biológicos) esobreos efeitos de programas de intervenção motora no desenvolvimento motor. Os resultados demonstraram que as intervenções nos primeiros anos de vida podem auxiliar nos ganhos do desenvolvimento humano e prevenir as incapacidades ou condições indesejáveis em crianças com atraso no desenvolvimento.

Vários estudos têm sido realizados verificando os efeitos de programas deintervenção motora, seja em crianças com desenvolvimento típico ou em crianças com atraso de desenvolvimento (VALENTINI; 2002; MISSIUNA, 2003; CANTELL; SMYTH; A HONEN, 2003; POETA; ROSA NETO, 2005; MÜLLER, 2008; WILLRICH et al., 2008; ROSA et al., 2008; PICK, 2008; MEDINA, 2008; CAMPOS et al., 2008; TSAI, 2009; BRAGA et al., 2009; CONTREIRA et al., 2010) demonstrando efeitos benéficos nas habilidades motoras na maioria dos casos em que foram utilizadas.

Os resultados deste estudo estão em conformidade com um estudo realizado por Valentini (2002), que teve como objetivo principal determinar a influência de uma intervenção motora no desenvolvimento motor e a percepção de competência física de crianças com atraso motor. Fizeram parte do estudo 91 crianças, na faixa etária entre seis e 10 anos, de ambos os gêneros, divididas em dois grupos distintos: Grupo Controle ( $G C$ - n=50) eGrupo Intervenção ( $G I$ - n=41). O grupo intervenção foi submetido a um programa de atividades motoras orientadas para a maestria com frequência de duas vezes por semana em um período de 12 semanas, com duração de 60 minutos cada sessão de intervenção motora. A autora obteve resultados estatisticamente significativos após a intervenção para o GI, para as habilidades decontrole de objetos, locomotoras ena percepção de competência física. Ainda, a autora reforça a importância da utilização de uma variedade deestratégias duranteas aulas queestimulam mel horias motoras, principal menteem crianças com atrasos motores que demonstram al gumas formas de fracasso escolar. Essas afirmações reforçam a efetividade do programa desenvolvido no presente estudo, que num período mais curto (10 semanas) evidenciou resul tados rel evantes na mel hora do desempenho motor dos escolares.

Poeta e Rosa N eto (2005) aval iaram o efeito da intervenção motora em uma criança com diagnóstico deTranstorno do Déficit deAtenção/ Hiperatividade (TDAH ). A avaliação motora foi realizada através da Escala de Desenvolvimento Motor - EDM (ROSA NETO, 2002) etambém foram aval iados outros aspectos por meio de um formulário biopsicossocial. A intervenção motora teve duração de 25 sessões duas vezes semanais. Considerando as dificuldades apresentadas por 
crianças com TDAH, que vão desde desordens motoras, escolares e em atividades diárias, os resultados revelaram que um programa de intervenção possibilitou mudanças positivas no equilíbrio corporal, motricidade fina, esquema corporal e organização temporal. Os resultados desta pesquisa confirmam a influência positiva da intervenção motora nos aspectos motores de crianças com dificuldade de movimento, o que reforça os resultados do presente estudo.

No estudo realizado por Tsai (2009) foi verificada a eficácia da intervenção no controle inibitório (percepção olho-mão) de crianças com TDC na prática de tênis de mesa por meio de uma abordagem ecológica. Quarenta e três crianças de nove a dez anos foram testadas usando a avaliação de movimentos MABC2, os participantes foram divididos em dois grupos, crianças com TDC $(n=27)$ e crianças típicas $(n=16)$. A s crianças com TDC foram submetidas a um programa de intervenção de 10 semanas de treino de tênis de mesa, com frequência de três vezes por semana. Os resultados demonstraram que a intervenção de exercício empregado em ambiente escolar causou melhorias significativas das capacidades cognitivas e funções motoras das crianças com TDC. O período de aplicação da intervenção assemelha-se ao presente estudo, confirmando que num período de 10 semanas é possível verificar mel horas motoras significativas.

Os efeitos de um programa de intervenção motora também foram investigados no estudo dePick (2008). Fizeram partedo estudo 76escolares, deambos os sexos, com idades entre 4 e 10 anos, comesem N ecessidades Educacionais Especiais divididas em Grupo Intervenção (crianças com e sem N ecessidades Educacionais Especiais e Grupo Controle (crianças com e sem NEE). Os participantes foram avaliados pela aplicação do T est of G ross M otor D evelopment - Second E dition (TGMD2). O programa deintervenção tevecomo objetivos a maestria einclusão social, num período de 14 semanas, aplicado a 35 crianças do GI. Dentre outros resultados encontrados observou-se que as crianças do GI apresentaram diferenças estatisticamente significativas nas habilidades de locomoção e controle de objetos após o programa, enquanto que as crianças do GC não apresentaram mudanças significativas nos pós-testes. Esses resultados corroboram os resultados da presente pesquisa, que também encontra no programa de intervenção motora uma estratégia benéfica para melhora na funcionalidade de crianças com atrasos motores.

Outro estudo que está em conformidade com as investigações anteriormentecitadas foi o realizado por Müller (2008). A autora aval iou a influência de um programa motor num período de 12 semanas (24 sessões), em diferentes contextos (individual domicílio, individual creche e grupo creche), para crianças na faixa etária entre seis e dezoito meses com atrasos motores. Como resul tados a autora encontrou diferenças estatisticamente significativas no desempenho motor das crianças nos diferentes contextos. A través desses resultados a autora reforça a relevância do desenvol vimento de intervenções motoras para crianças com atrasos motores e salienta que quanto mais cedo for diagnosticado o atraso motor, mais cedo também podem ser elaboradas propostas interventivas a fim de potencializar o desenvolvimento. 
A fim de identificar o perfil motor de crianças de baixo nível socioeconômico e verificar o efeito de um programa de intervenção psicomotora, Campos et al. (2008), avaliaram seis crianças do gênero masculino, na faixa etária de dez a doze anos. Os autores utilizaram uma bateria psicomotora avaliando tonicidade, equilibração, lateralização, noção do corpo, estruturação espaçotemporal, praxia global efina. Com basenas dificul dades detectadas foi elaborado um programa de intervenção psicomotora, aplicado durante três meses, com 16 sessões de uma hora de duração, duas vezes por semana. A pós o programa de intervenção houveaumento estatisticamentesignificativo na pontuação dos fatores tonicidade, equilibração, estrutura espaço-temporal, praxia global efina nas crianças baixo nível socioeconômico, o que vem a confirmar a importância de programas de intervenção para o desempenho psicomotor. Observando-se o período de desenvolvimento do programa citado nota-sea relevância do presenteestudo, que com duração de 10 semanas e 45 minutos por sessão foi eficaz na mel hora motora das crianças.

Outro estudo interventivo foi realizado por Braga et al. (2009), com o objetivo de investigar a influência da intervenção motora no desempenho das habilidades motoras decrianças com idade ente seis e seteanos. Participaram desse estudo 60 escolares, do gênero masculino e feminino, distribuídos em três grupos com avaliação do desempenho das habilidades locomotoras através da bateria de testes TGMD-2. Os escolares participaram da intervenção no período de 12 semanas e os resultados ratificam a influência positiva do programa de intervenção, demonstrando uma diferença significativa nas médias dedesempenho dos grupos do pré para o pós-teste.

Já nos estudos realizados por Medina (2008) e Contreira et al. (2010), ambos utilizando intervenção motora, não foram encontrados resultados efetivos após os programas. N o primeiro estudo foi observado o efeito das dicas na aquisição do rolamento peixe em crianças com TDC e crianças típicas. As sessões foram compostas de sete aulas no período de três semanas, em dias alternados. Houve aumento dos escores do pré para o pós, contudo não ocorreu diferença estatisticamente significativa após as sessões. A autora acredita que as dicas utilizadas não foram específicas o suficiente para gerar modificações significativas na aprendizagem do rolamento peixe, tendo em vista o nível do desenvolvimento motor dos participantes.

No estudo de Contreira et al. (2010) as autoras verificaram os efeitos de um programa de natação e ginástica respiratória no estilo de vida e desempenho motor de crianças e adolescentes asmáticos. Cabe ressaltar que crianças com problemas respiratórios tendem a apresentar atrasos no desenvolvimento motor pelo fato denão seenvolverem ativamenteem práticas motoras devido às suas condições desaúde. As autorasnão obtiveram resultados demelhora motora após a intervenção motora em meio aquático e atribuem esses resultados ao não desenvolvimento de atividades voltadas para as dificuldades motoras apresentadas pel as crianças, o que 
difere do presente estudo que encontrou melhoras significativas no desempenho motor dos escolares por realizar atividades motoras específicas.

Com basenos resultados obtidos no presenteestudo eem conformidade com as investigações anteriormente citadas que obtiveram resultados efetivos em relação aos aspectos motores por meio de programas de intervenção motora, constatou-se que um programa de intervenção com fundamentação desenvolvimentista foi eficiente para estimulação das habilidades motoras das crianças com indicativo de TDC no grupo estudado.

Em relação ao desempenho das crianças com indicativo de TDC por categoria dehabilidademotora, esteestudo apresentou como resultados um mel hor desempenho na habilidade de equilíbrio, seguida dehabilidades delançar ereceber e destrezas manuais.

No estudo de Silva (2009), realizado com 406 escolares de ambos os sexos, de uma escola Municipal de São José/ SC, foi avaliado, dentre outras variáveis, o desempenho motor através do MABC-2. Os resultados obtidos revelaram que os escolares apresentaram maiores médias nas habilidades de destrezas manuais, seguidas de equilíbrio e habilidades com bola. É importante destacar que não há um consenso estabelecido na literatura sobre em quais as habilidades as crianças com indicativo de TDC têm mel hor domínio, o que auxilia no entendimento dos resultados distintos entre os estudos discutidos.

Ao investigar as principais características das crianças de nove a dez anos de idade com indicativo de TDC, Pellegrini et al., (2006) avaliaram o desempenho motor por meio do MABC. Participaram do estudo 111 meninos e 135 meninas, de uma escola pública. Os resultados indicaram que 26 das crianças foram identificadas com TDC, sendo 21 meninas ecinco meninos, quanto ao escore por categoria do MABC, os resultados demonstraram que as tarefas de equilíbrio foram executadas com sucesso por quasetodas as crianças, porém com porcentagem aproximada de $10 \%$ das crianças encontrou dificuldade na categoria habilidades com bola e, aproximadamente, $15 \%$ delas apresentou sérias dificuldadena categoria destreza manual, as autoras sugerem que essas diferenças no desempenho de habilidades motoras podem estar relacionadas às influencias sócio-culturais.

Estil, Ingvalsem eWhiting (2002) avaliaram crianças por meio do MABC com o objetivo deverificar o desempenho espacial etemporal, esseestudo envolvia dois grupos de crianças com 10 anos de idade. Em um dos grupos as crianças tinham problemas de coordenação de movimentos (TDC), enquanto o outro grupo (controle) era constituído por crianças com desenvol vimento típico. Os participantes foram avaliados em uma anál ise cinemática em um ambiente de laboratório e os resulltados revelaram que houvediferenças significativas entreos grupos, em favor do grupo controle, com relação tanto ao desempenho espacial quanto ao temporal na interceptação da bola em movimento. As crianças com TDC iniciaram o movimento de apanhar a bola mais tardiamente e o movimento de preensão dos 
dedos antes do tempo em relação ao grupo controle.

No estudo de Miranda (2010) foi analisado o perfil motor de escolares, de ambos os sexos, na faixa etária entre os setee 10 anos, estudantes de escolas da rede pública da região continental de Florianópolis/ SC. A autora evidenciou que os escolares apresentaram um pior desempenho nas habilidades de destreza manual, enquanto que nas habilidades de equilíbrio e lançar e receber obtiveram um melhor desempenho. Já França (2008) em seu estudo objetivou caracterizar o desempenho motor ea prevalência de TDC em 417 escolares, de ambos os sexos, nafaixa etária deseteeoito anos da redepública deFlorianópolis/ SC. Os resultados deste estudo foram semel hantes aos de Miranda (2010), com desempenho pior nas habilidades de destreza manual.

A pesar dos resultados distintos entre os estudos observa-se que os escolares com indicativo de TDC apresentam dificuldades no desempenho de habilidades finas e amplas. Já no presente estudo verificou-se que crianças com indicativo deTDC apresentam maiores prejuízos nas habilidades dedestreza manual. Diante disso, acredita-se que as crianças devem ser estimuladas por meio de atividades escolares e domiciliares, para que possam ter autonomia na realização destas atividades, superando, desse modo as dificuldades do dia a dia. Logo, tal iniciativa propicia, inclusivea integridadee o bem-estar decrianças em idadeescolar.

\section{CONCLUSÃo}

Os resultados do presente estudo revelaram que o programa de intervenção motora proposto foi efetivo para a mel hora do desenvolvimento motor das crianças com indicativo deTDC. Esteestudo confirmou sua rel evância científica ao demonstrar o valor dos movimentos na vida das crianças com indicativo de TDC ao estimular as capacidades de seus corpos, experimentando movimentos diversificados que podem estar presentes nas atividades diárias ou esportivas.

Desse modo, destacamos a necessidade e a importância do desenvolvimento de projetos dessa natureza, a fim de oportunizar e potencial izar as habilidades dos escolares com dificuldades motoras.

\section{Referências}

BRAGA, R. K. et al. A Influência de um programa de intervenção motora no desempenho das habilidades locomotoras de crianças com idade entre 6 e 7 Anos. Revista da Educação Física / U EM. Maringá, v. 20, p. 171-181, jul. 2009. Disponível em: http:/ / www.periodicos.uem.br/ ojs/ index.php/ RevEducFis/ article/ view/6133/4427>. Acesso em: 13 jul. 2010. 
CANTELL, M. H.; SMYTH, M. AHONEN, T. P. Two distinct pathways for developmental coordination disorder: Persistence and resolution. H uman M ovement Science. Department of Paediatrics, University of Cal gary, 2003, p. 413 - 431.

CAMPOS, A . A. A daptação cultural da escala "perfil de auto-percepção para crianças". Dissertação (Mestrado em Ciências do Desenvolvimento Humano) - Universidade do Rio de Janeiro. Rio de Janeiro, 2004.

CAMPOS, A, C. et al. Intervenção psicomotora em crianças denível socioeconômico baixo. Revista Fisioterapia e Pesquisa, v.15, n.2, p.188-93, 2008.

CERMAK, S. A. Developmental dyspraxia. In: ROY E. A. (Ed.). N europsychological Studies of A praxia and Related D isorders. A msterdam: North Holland; 1985. p.225-248.

CONTREIRA, A. R. et al. O efeito da prática regular de exercícios físicos no estilo de vida e desempenho motor de crianças e adolescentes asmáticos. Pensar a Prática, Goiânia, v.13, n.1, p.1-17, 2010.

ESTIL, L. B.; INGVALSEN, R. P.; WHITING, H. T. A. Spatial and temporal constraints on performance in children with movement co-ordination problems. Exp Brain Res, v.147, p.153-161, 2002.

FERREIRA, F. L. et. Desordem da coordenação do desenvolvimento. 2006, M otriz, Rio Claro, set/ dez. 2006. Disponível em: http:/ / www.cecemca.rc.unesp.br/ ojs/ index.php/ motriz/ article/ viewFile>. A cesso em:18 out. 2009.

FRANÇA, C. Desordem coordenativa desenvolvimental em crianças de 7 e 8 anos de idade. Dissertação de Mestrado (Ciências do Desenvolvimento Humano) - Universidadedo Estado de Santa Catarina. Florianópolis, 2008.

GALLAHUE, D. L., DON NELLY, F. E du cação física desenvolvimentista para todas as crianças. 4. ed. São Paulo: Porte, 2008.

GALLAHUE, D. L.; OZMUN, J. C. Compreen dendo o desenvolvimento motor: bebês, crianças, adolescentes e adultos. 3. ed. São Paulo: Phorte, 2005.

GEUZE, R. H. et al. Clinical and research diagnostic criteria or developmental coordination disorder: a review and discussion. H uman M ovement Science, Amsterdam, v. 20, p.7-47, 2001.

HENDERSON. S. E.; SUGDEN, D. A. BARNETT. A. L. M ovement A ssessment Battery for Children-2: Examiner's M anual, Second Edition (M ovement A BC-2). Pearson Assessment, 2007.

JORGE, M. R. M anual diagnóstico e estatístico de transtornos mentais. DSM -IV -TRTM . 4. ed. Tradução Cláudia Dornelles. Porto Alegre: Artmed, 2003.

LEMOS, E., FRACAROLI, M. N., ROSA, O. A. Crianças “descoordenadas são também crianças com baixo nível de aptidão física? São Paulo: Escola deA rtes, Ciências eH umanidades/ USP. Disponível em: HTTP:/ / www.naeg.prg.usp.br/ pep07/ arquivos/ 565/ ECP_CYH_2008.pdf>A cesso em: 02 nov. 2009.

MISSIUNA, C. Children with Developmental Coordination Disorder: At home and in the Classroom. Ontário, Canadá: CanChild, Centre for Childhood Disability Research, 2003.

MULLER, A. B. Efeitos da Interven ção motora em diferentes contextos no desenvolvimento da criança com atraso motor. Dissertação (M estrado) - UniversidadeFederal do Rio Grande do Sul, 2008. 
MEDINA, J. et. al. O efeito de dicas deaprendizagem na aquisição do rolamento peixe por crianças com TDC. Revista Brasileira de Ciência, Campinas, v. 29, n. 2, p. 79-94, jan. 2008.

MIRANDA, T. B. Perfil motor de escolares de 7 a 10 anos de idade com Indicativo de $D$ esordem Coordenativa Desenvolvimental (DCD). Dissertação (Mestrado em Ciências do Movimento Humano) - Universidade do Estado de Santa Catarina, 2010.

PELLEGRINI, A. M.; et al. Dificuldades motoras em crianças de9-10 anos de idade: seriam os meninos mais descoordenados? Julh. 2006. p. 77-88. Disponível em: www.unesp.br. A cesso em: 16 jun. 2010.

PEREIRA, K. SILVA, L. CAMPOS, A. C. Intervenção psicomotora em crianças de nível socioeconômico baixo. Rev. Fisioterapia e Pesquisa, São Paulo, v. I5, n.2, p.188-93, 2008.

PICK, R.K. Influência deum programa deintervenção motora inclusiva no desenvolvimento motor e social de crianças com atrasos motores. Dissertação (Mestrado em Ciências do Movimento Humano) - Universidade do Estado do Rio Grande do Sul - UFRGS, 2008.

PIFFERO, C. M. H abilidades motoras fundamentais e especializadas, aplicação de habilidades no jogo e percepção de competência de crianças em situação de risco: a influência de um programa de intervenção de iniciação ao tênis. Dissertação (M estrado) - UniversidadeFederal do Rio Grande do Sul, Porto Alegre. 2007.

POETA, L. S. A valiação motora e intervenção motora em escolares com indicadores de transtorno do déficit de aten ção/hiper atividade(TD A H ). Dissertação (Mestrado em Ciências do M ovimento Humano) - Universidade do Estado de Santa Catarina, 2005.

POETA, L. S; ROSA NETO, F. Intervenção motora em uma criança com transtorno de déficit de atenção/ hiperatividade (TDAH). Lecturas Educación Física y D eportes, v.10, n.89, 2005. Disponível em: http:/ / www.efdeportes.com. Acesso em: 02 fev. 2010

ROSA NETO, F. M anual de avaliação motora. Porto Alegre: Artmed, 2002.

ROSA, G. K. et. al. Desenvolvimento motor de criança com paralisia cerebral: avaliação e intervenção Rev. Bras. Ed. Esp., Marília, 2008, v. 14, n. 2, p. 163-176.

RUIZ, L.M., et al. The assessment of motor coordination in children with the Movement A BC test: a comparative study among Japan, USA and Spain. International Journal of Sport Science, v. 15, n.1, p. 22-25, 2003.

ROSA , O. A . Crianças "Descoordenadas" são também crianças com baixo nível de aptidão física?: em foco o nível de aptidão física de crianças com dificuldades motoras. Escola de Artes, Ciências eH umanidades/ USP. 2009. Disponível em: http:/ / www.naeg.prg.usp.br/ pep07/ arquivos/ 565/ ECP_CYH_2008.pdf>A cesso em: 02 nov. 2009.

SANTOS, S., DANTAS, L., OLIVEIRA, J. A. Desenvolvimento motor decrianças, de idosos edepessoas com transtornos da coordenação. R evista Paulista de E ducação Física, São Paulo, v. 18, p.33-44, 2004. Disponível em: http:/ / www.usp.br/ eef/ rpef/ v18esp70anos/ v18p33.pdf>. Acesso em: 05 nov. 2009.

SILVA, J. 2009. D esempenho motor, desempenho acadêmico esenso de auto-eficácia de escolares do ensino fundamental. Dissertação (Mestrado em Ciências do Movimento Humano) Universidade do Estado de Santa Catarina, Florianópolis, 2009. 
THOMAS, J.; NELSON, J. M étodos de pesquisa em atividade física e saúde. 3. ed. São Paulo: Artmed Editora, 2002.

TSAI, C. L. The effectiveness of exercise intervention on inhibitory control in children with developmental coordination disorder: Using a visuospatial attention paradigm as a model. Rev. Research in D evelopmental Disabilities, v.30, n.6, p.1268-1280, 2009.

VALENTINI, N. V. A influência de uma intervenção motora no desempenho motor e na percepção de competência de crianças com atrasos motores. R evista Paulista de Ed. Física, São Paulo. p.61-75, 2002.

WILLRICH, A. et. al. Desenvolvimento motor na infância: influência dos fatores derisco e programas de intervenção. R evista N eurociência, 2008. 